\title{
Early life secondhand smoke exposure assessed by hair nicotine biomarker may reduce children's neurodevelopment at 2 years of age
}

\begin{abstract}
Exposure to secondhand smoke (SHS) can affect fetal brain development as well as subsequent neurodevelopment. This study aimed to determine the association between prenatal and postnatal SHS exposure with children's neurodevelopment at 2 years of age. Among 107 mother-child pairs from a Malaysia prospective cohort, prenatal and postnatal SHS exposure was determined based on maternal and child hair nicotine concentrations. Multiple linear regressions were used to determine the association between prenatal and postnatal levels of nicotine in maternal and children's' hair with children's neurodevelopment. After adjustment for confounders, prenatal nicotine concentration levels were negatively associated with communication $(\beta=-2.059 ; p=0.015)$ and fine motor skills $(\beta=-2.120 ; p=0.002)$ while postnatal nicotine concentration levels were inversely associated with fine motors $(\beta=-0.124 ; p=0.004)$ and problem solving skills $(\beta=-0.117 ; p=0.013)$. In conclusion, this study suggests that early life exposure to SHS may affect children's neurodevelopment.
\end{abstract}

Keyword: Secondhand smoke; Prenatal; Postnatal; Hair nicotine; Neurodevelopment; Children 